\title{
Integration of Dynamic Tariff Scheme in Congestion Control
}

\author{
Ameya Patil \\ Student \\ Shree L. R. Tiwari \\ College of Engineering \\ Mira road, India
}

\author{
Aditya Badve \\ Student \\ Atharva College of Engineering \\ Mumbai, India
}

\begin{abstract}
Due to large numbers of increasing amount of customers, the congestion in cellular networks during summit hour and at hot spot areas is a very serious problem which is faced by almost every cellular company and their customers mainly in large metropolitans now a days. Solution of this congestion is very easy and straightforward as it can be resolve by enhancing and upgrading the infrastructure. However, this is not an economical solution as large amount of investment is involved in it. Many service providers tempt to avoid it. However, even after resolving such issue, the traffic requests are increasing enormously and the problem of congestion remains forever. This is very serious problem in view of customers and the service providers and it is need to be address immediately. This Proposed program tries to address the previously mentioned problem of congestion in cellular networks by introducing a new idea of call duration control coupled with dynamic tariff and Hold-The-Call principle. In addition, it proposed to introduce a new concept called Guard Channel Scheme and Priority Queue Scheme along with clustering techniques such as K-bisecting algorithm to increase the efficiency. Proposed algorithm is being utilize to restrict the duration of calls depending upon current traffic conditions. In dense traffic conditions, the network restricts the duration of ongoing call up to the particular time limit beyond which the customer has to pay higher tariff to continue the call. At the same time the principle of the Hold-The-Call can also be apply so that if a recently generated call does not get a traffic channel then it should not be blocked but may put on hold in a queue (Priority queue) to get a traffic channel at earliest. This program will help to reduce the level of congestion significantly without compromising with system performance. At the same time, it will marginally increase the revenue per unit time.
\end{abstract}

\section{Keywords}

Congestion control, Dynamic tariff, Guard channel scheme, Priority queue scheme, and K-bisecting algorithm

\section{INTRODUCTION}

The users of cellular phones are tremendously increasing at a continuous rate. So the problem of congestion is becoming more and more critical. Since the infrastructure capacity is not increased to that proportion, the rise of call dropping and call blocking probability especially during peak hours and at the hot spots is also increased intolerably. In the past, some solutions have already been suggested to resolve this problem. In addition, it has been also observed that the networks become unreachable due to exceptionally high traffic at critical times like natural or manmade disasters or calamities. Therefore, there is an urgent need to find out a solution, which will reduce the call blocking as well as the call dropping probability without compromising with the system utilization. This problem can be resolve easily by using 'Dynamic tariff Scheme' where the tariff will decided on base of the traffic conditions in the cell area. It will put the financial burden on users who want to call from the network, which is already been in the heavy traffic conditions. Because of the higher tariff charges for the entire duration of the call, some user may drop their calls by themself.

Dynamic tariff scheme will reduce the probability of the call dropping, call blocking and will increase the revenue of the service providing company. However, to implement this algorithm dynamic tariff have to add an additional mechanism that can reduce the time complexity. Therefore, this scheme is introducing K-bisecting mechanism to reduce the searches made during Dynamic tariff scheme implementation.

\section{LITERATURE SURVEY AND RELATED WORK}

Call-level control is defined as a set of actions, performed at call set-up phase, to determine whether or not the resources requesting the call can be accepted. It tries to prevent congestion by not allowing new calls into the network unless the network has sufficient capacity to support them. An example of the call-level control is Call Admission Control (CAC).

\subsection{Call Admission Control (CAC)}

The major design concern of CAC is to prioritize handoff calls, because mobile users tend to be much more sensitive to call dropping than to call blocking. Various handoff priority-based CAC schemes have been proposed [10], which can be classified into two broad categories: Guard Channel Schemes, Queuing Priority Schemes. Describing them as following.

\subsection{Guard Channel Scheme}

A Guard Channel Scheme proposed to priori assign a higher capacity limit for handoffs than for new calls. The difference between these two limits is called 'Guard Channel'. This scheme reserves certain number of channels in the given access point exclusively for handoff calls. A new call request will admitted if the only remaining resources can accommodate the requested bandwidth.

\subsection{Priority Queue Scheme}

In this scheme, calls are accepted whenever there are free channels. When all channels are busy, new calls will be queued while handoff calls are blocked, new calls are 
blocked while handoff calls are queued, or all arriving calls are queued with certain rearrangements in the queue.

\subsection{K-bisecting Algorithm}

By clustering we will understand grouping terms, documents or other items together based on some criterion for similarity. K-bisecting algorithm is a combination of $\mathrm{K}$ means and hierarchical clustering. It starts with all objects in a single cluster. Here, this technique is used to improve time complexity. [11]

\section{REQUIREMENT ANALYSIS \\ 3.1 Problem Definition}

One of the major problem is now being faced by cellular network providing companies is the congestion over the cellular network. This congestion might be Damage Company's reputation among the consumers. As the numbers of customer are increasing at a rapid rate, the problems of network congestion, call holding and call dropping are becoming more critical. Since the upgradation in infrastructure does not allow due to economical limitation to resolve this problem, service providing companies may be come up with more reliable and cost effective solutions to control the problem. When the call arrival rate is momentarily very high (for example in peak hours), no matter how the parameters are adjusted, these existing schemes cannot ensure the QoS (Quality of Service) to consumers. The main reason of degradation of QoS stems from the fact that resources in a wireless network, such as timeslots, code and power, are shared by all users. One user's interference into the network will reduce QoS of the other users in that same network. So, the observation proves that the congestion in the network cause the QoS degradation. However, the current CAC schemes cannot avoid congestion. Because they do not provide incentive for users to use the channel resources effectively. In broadband networks, pricing schemes are widely discussed as means for traffic management and congestion control. Through pricing, the network can send signals to the users, providing incentive that influences their behavior. This provides another dimension for the design of CAC schemes that can be used in wireless networks as well. In this proposed scheme integrate dynamic tariff with CAC to address the problem of congestion.

Many alternatives solutions been already discussed in the literature part. But none of them are efficient for handling call dropping, network congestion and call blocking. Network providing companies has limited bandwidth and frequency spectrums. But demands for mobile communication services are increasing rapidly. So it is very important for these companies to use these limited bandwidth and frequency spectrums very efficiently. In recent years many congestion control algorithm have primarily focused on the Channel Allocation and Call Admission Control (CAC) problems. These schemes have been also proposed in literature. In order to handle congestion in the network and provide Quality of Service (QoS) to the consumers, Call Admission Control (CAC) is been used. Call Admission Control (CAC) limits the number of call connections into the networks. Call blocking probability and handoff call blocking probability are two important part of the Quality of Service (QoS). Handoff calls are usually given a highest priority because the call is forced to terminate during the service is more irritating than a call is being blocked at time of it start. Various handoff priority based CAC schemes have been proposed in literature including Guard Channel Schemes, Queuing Priority Schemes and Channel Borrowing Schemes. These schemes have focused on how to adjust the trade-off between call blocking probability and handoff call blocking probability. Those schemes can improve the system performance if call arrival rate is within a certain dynamic range. However, these result which confer by these research states that if the call arrival rate is increased then both the call blocking probability and the handoff call blocking probability get increased respectively. When the call arrival rate is temporarily very high (e.g. in busy hours), no matter how the parameters are adjusted, these schemes cannot guarantee the Quality of Service(QoS) to consumers. The resources called timeslots, code and power, are shared by all the users in the wireless network. This is the main reason behind the degradation of the Quality of Service (QoS). When one user is entered into the network, it will cause QoS degradation to other users within the same network. In general the most serious Quality of Service(QoS) degradation occurs during the congestion. However, the current Call Admission Control(CAC) schemes are not able to avoid congestion. Because they do not provide incentive for users to use the channel resources effectively. In broadband networks, pricing schemes are widely discussed as means for traffic management and congestion control. Through pricing, the network can send signals to the users, providing incentives that influence their behavior. This provides another dimension for the design of CAC schemes that can be used in wireless networks as well. In this thesis, dynamic tariff is integrated with CAC to address the problem of congestion. [1] [8]

\subsection{Scope}

Proposed scheme shows a substantial reduction in call blocking as well as call dropping probability without comprising with the system utilization. In fact there is a marginal rise in the revenue generated per unit time as compared with existing schemes. Thus it takes into consideration the service provider's viewpoint as well. This is a very important feature of the proposed scheme making it commercially viable for implementation. Average delay in establishment of a call within a tolerable range will be preferred by the customer than a total rejection of a call. Some users may not like the concept of call termination by the network. These users at large will prefer this scheme once they find that there is a substantial reduction in call blocking probability. Thus, in this scheme a new comprehensive call management strategy, which tries to distribute the available resources in a more equitable manner ensuring fair treatment to all the users. At the same time the scheme is also flexible which allows a user to utilize the resources without any limit by paying at a higher tariff. There are many other schemes, which are also proposed by the researchers. However, the service providing companies have not yet selected and utilized any of the schemes. The cellular traffic is continuously increasing and the corresponding expansion of the infrastructure is not being increased to its capacity. Then such situations like congestion are always bound to occur when the traffic demands temporarily increases than the capacity of the system. In such situations there will be a need of an effective strategy to deal with this situation and this proposed scheme can be embedded in existing setup. 


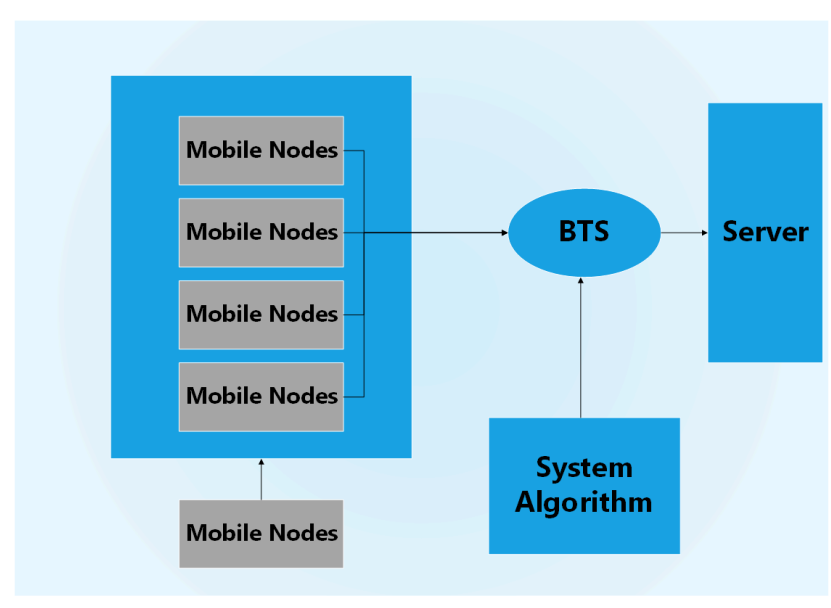

Figure 1: Block diagram

\section{METHODOLOGY USED}

This scheme is introducing a new algorithm called Dynamic tariff. This algorithm tried to control the congestion in the network. It is very clear that the problem of congestion can be solved with two approaches. One of the natural solutions appears to be an increase in the network capacity with more base stations. However, it requires huge infrastructure investment, which is not immediately viable everywhere. Also it is not economically viable because the capacity of the system is not then fully utilized at the off-peak hours. Therefore, it is very obvious to find out an another solution.

The congestion can also be reduced if the traffic demand is reduced by some means. The traffic demand is proportional to the call arrival rate and average call duration. Therefore if the average call duration is reduced then it will naturally reduce the traffic demand and will solve the problem of congestion up to the satisfactory level. Proposed solution tries to adopt the aforesaid approach. The simulations carried out show a very remarkable improvement in a call blocking probability. The scheme can be easily implemented in the existing base station setups of the service providers. Also it will not adversely affect the system utilization and revenue generation. This scheme needs to be worked out such that the users are discouraged to continue the non-urgent communication beyond certain duration at the time of heavy traffic demand. Instead, customers can continue with such communication at other times (e.g. at off-peak hours). This can be achieved by effective call management strategy given by dynamic tariff. This proposed scheme, tries to address the problem of congestion with the following approach.

Proposed system is using Dynamic Tariff Scheme, Call Admission Control, guard channel scheme, along with the clustering algorithm and scheduler logic to enhance the efficiency of the algorithm. This approach is to add the calls to channel until the congestion state is achieved. After this, using "K-bisecting" algorithm for dividing channels into various groups according to their usage. During the congestion state, it measures the status of channel after every ' $t$ ' seconds. This called a scheduler logic. Scheduler logic is used to decide the criticality of the network status. According to the level of congestion, dynamically tariff will apply to the consumers. These tariff data are stored in the price database. If congestion in network is very critical then highest tariff will be applied to the respective consumers and if congestion occurs but its state is not so critical, then price should be little higher than normal tariff but lower than higher tariff. As per the criticality status, select dynamic tariff scheme to increase the revenue and provide better network for maintaining the QoS by using channel borrowing. At bottleneck situation, using of channelborrowing scheme will help service provider to provide better cellular network to users. With the help of scheduler logic, network state is continuously getting monitored, so customer will pay additional charges only when the network state is in congestion. This scheme will reduce the call drop and will increase the revenue than any other previous schemes.

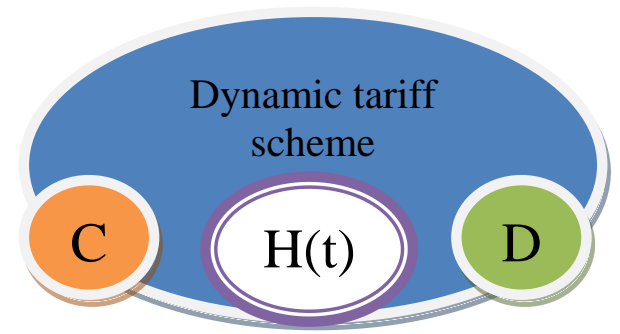

Figure 2: The implementation

In Figure 2, there are three major components such as $\mathrm{C}, \mathrm{H}$ $(t)$, D. C stands for Clustering function, H ( $t$ ) stands for transfer function of tariff block, D stands for Dynamic function.

\subsection{Flow chart}

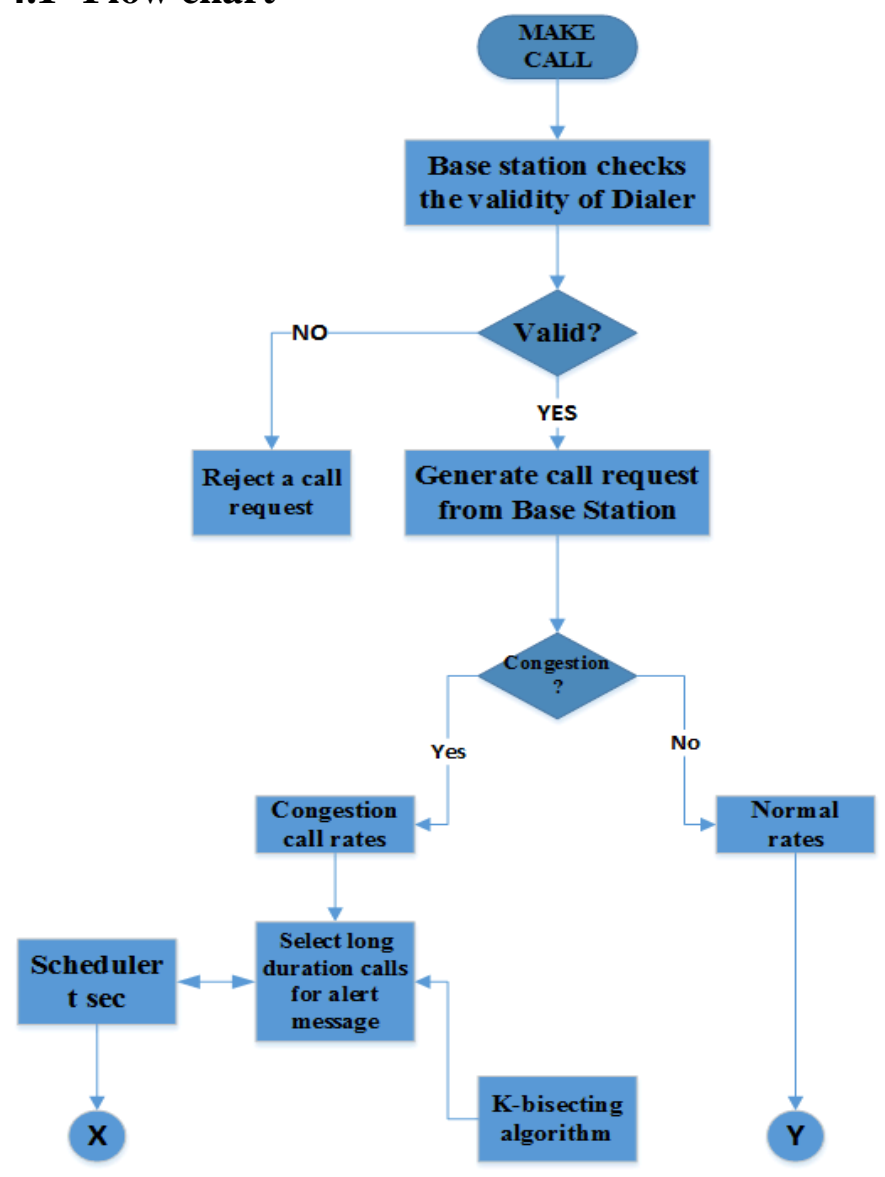




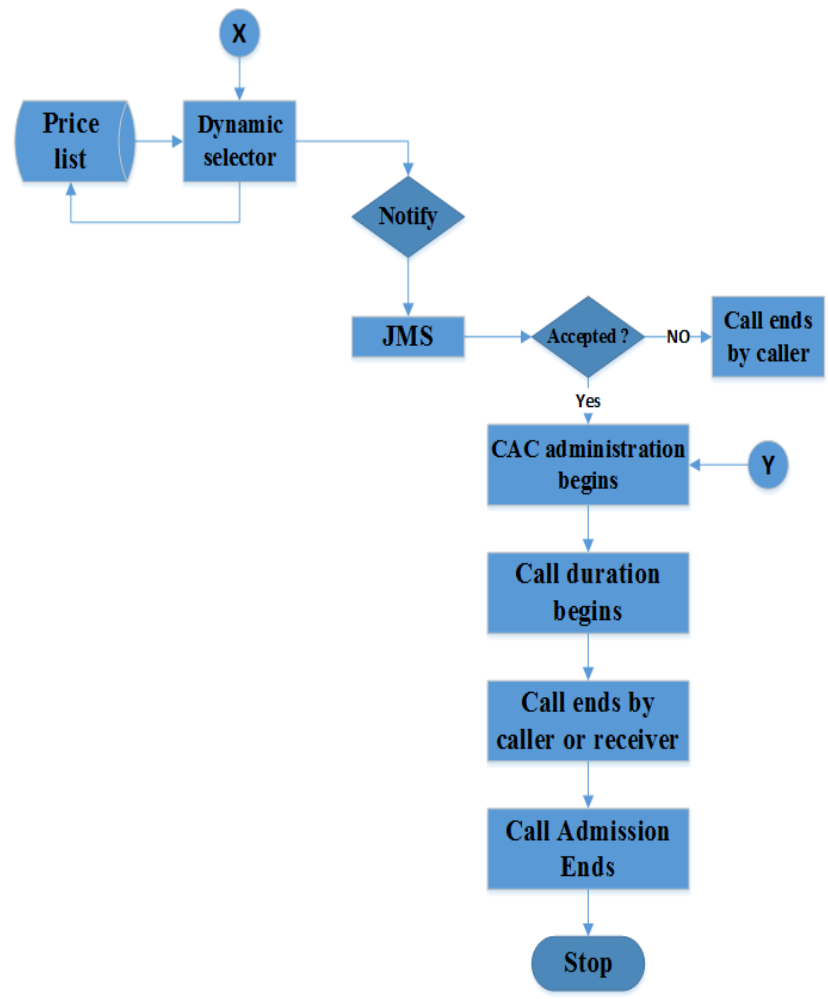

Table 3: Revenue Generation

\begin{tabular}{|l|l|c|c|c|c|c|}
\hline $\begin{array}{c}\text { Call } \\
\text { Arrival } \\
\text { Rate }\end{array}$ & \multicolumn{6}{|c|}{ Revenue per unit time(S) } \\
\cline { 2 - 7 } & $\begin{array}{c}\text { Normal } \\
\text { method }\end{array}$ & $\begin{array}{c}\text { Case } \\
1\end{array}$ & $\begin{array}{c}\text { Case } \\
2\end{array}$ & $\begin{array}{c}\text { Case } \\
3\end{array}$ & $\begin{array}{c}\text { Case } \\
4\end{array}$ & $\begin{array}{c}\text { Case } \\
5\end{array}$ \\
\hline 600 & 29.74 & 29.74 & 29.66 & 29.75 & 29.7 & 29.47 \\
\hline 700 & 33.53 & 33.78 & 33.81 & 33.58 & 34.1 & 33.66 \\
\hline 800 & 36.21 & 36.51 & 36.92 & 36.54 & 37.2 & 36.51 \\
\hline 900 & 37.67 & 38.34 & 38.91 & 38.36 & 39.1 & 38.47 \\
\hline 1000 & 38.35 & 39.44 & 40.50 & 39.53 & 41.0 & 39.66 \\
\hline 1100 & 38.85 & 40.58 & 41.88 & 40.74 & 42.1 & 40.80 \\
\hline 1200 & 39.07 & 41.44 & 42.81 & 41.54 & 42.4 & 41.42 \\
\hline
\end{tabular}

\subsection{Analysis of Simulation Results}

Result analysis graph of Current Scheme

With 900 users 120 seconds

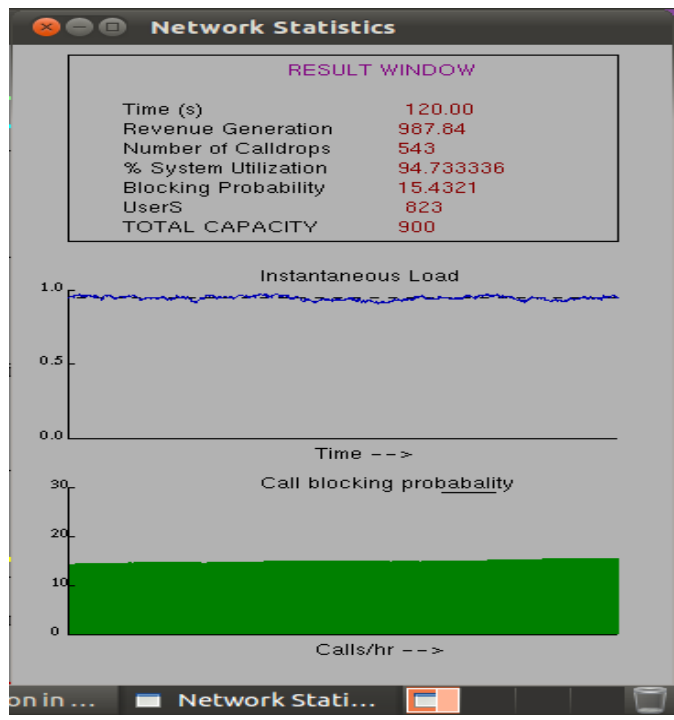

Result analysis graph of Proposed Scheme

With 900 users 120 seconds

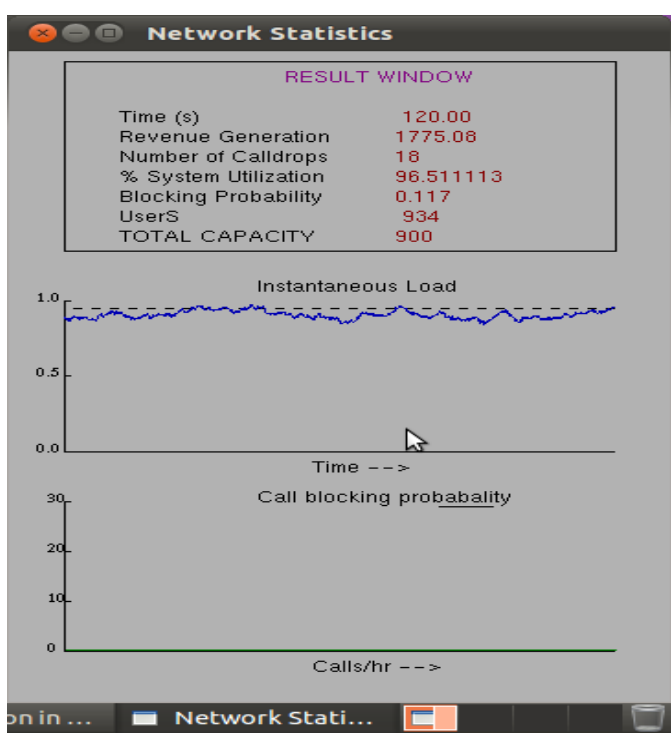


Result analysis graph of Current Scheme

With 1200 users 60 seconds

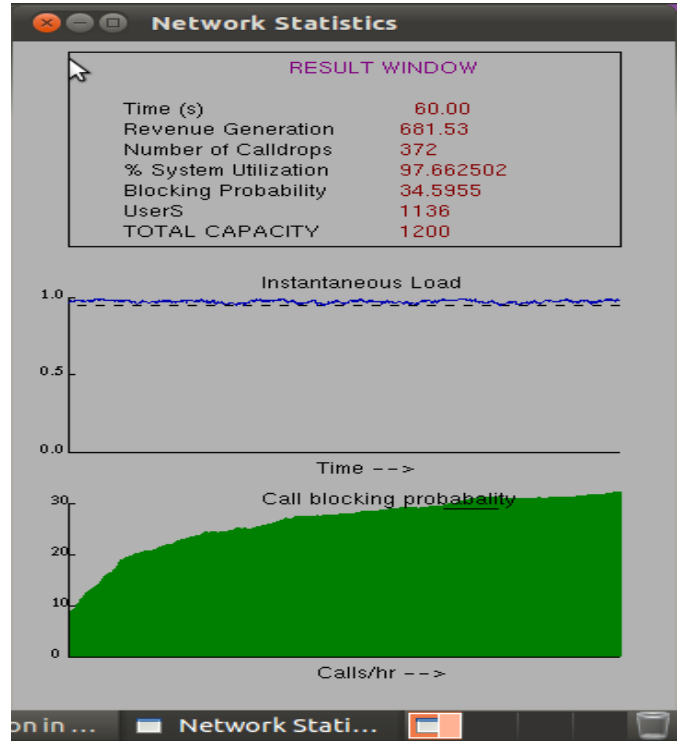

Result analysis graph of Proposed Scheme

With 1200 users 60 seconds

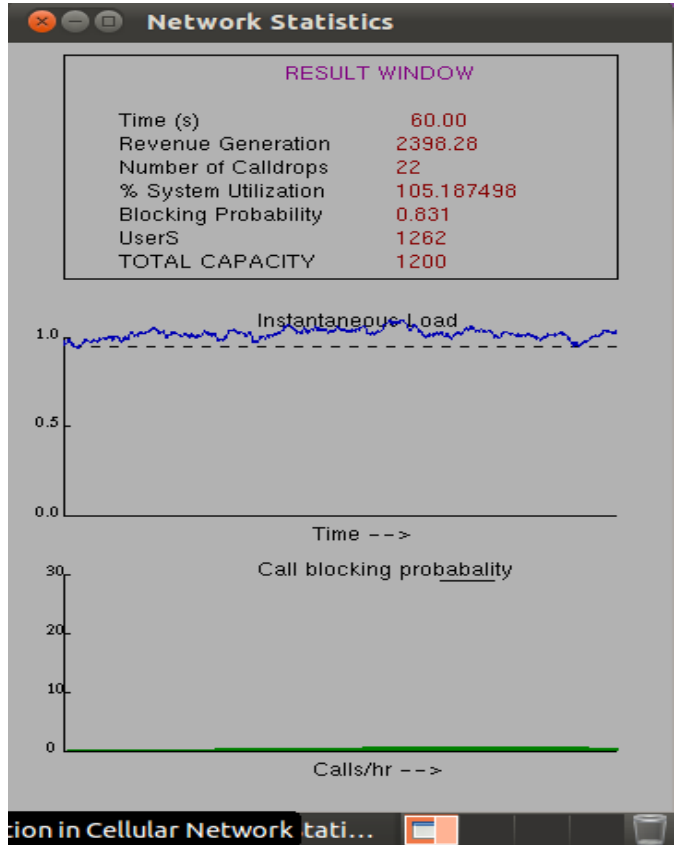

\section{CONCLUSION AND FUTURE SCOPE}

From the Simulation results and tabular results analysis, it is very clear that proposed scheme shows substantial reduction in call blocking probability without compromising with the system utilization. In fact, there will be a marginal rise in the revenue generated per unit time as compared with existing method. Thus, it also considers the service provider's viewpoint as well. This is a very important feature of the proposed scheme, which makes it commercially viable for implementation. Many users may not be agreeing with call termination by network concept. For that proposed scheme has an additional feature that will enhance the performance of call termination concept. Addition of clustering, scheduler and channel borrowing concept network will help in the bottleneck situation. Certainly, it will give the better performance during congestion and increase in revenue of the service provider. The special feature of this scheme is that the scheme is very flexible and allows the user to utilize the resources without any limit by paying at a higher tariff. Selection of a particular case of the proposed scheme is to be decided by the service provider.

According to this analysis, the cellular traffic is exponentially increasing and at the same time the infrastructure is increasing very linearly. Due to this the congestion in network is bound to be occur. In such situations, there is an urgent need of an effective strategy to deal with the crisis. This proposed scheme provides an effective solution, which can be easily implemented with the existing setup. In future proposed scheme can be modified by changing the algorithms implemented to give effective performance to cope up with the increasing cellular network traffic.

\section{REFERENCES}

[1] R. Cocchi, S. Shenker, D. Estrin and L. Zhang, "Pricing in Computer Networks: Motivation, Formulation and Example," IEEDACM Transactions on Networking, Vol. 1, No. 6, December 1993.

[2] P. C. Fishbum and A. M. Odlyzko, "Dynamic Behavior of Differential Pricing and Quality of Service Options for the Internet," ICE'98, pp. 128-139

[3] J. Hou and Y. Fang, "Mobility-based Channel Reservation Scheme for Wireless Mobile Networks," Proceedings of ZEEE WCNC 2000, Chicago, September 2000.

[4] H. Ji, J. Y. Hui and E. Karasan, "GoS-Based Pricing and Resource Allocation for Multimedia Broadband Networks," Proceedings of IEEE INFOCOM 1996, pp. 1020-1027.

[5] K. R. Krishnan, 'The Convexity of Loss Rate in an Erlang Loss System and Sojourn in an Erlang Delay System with Respect to Arrival rate and Service Rate," ZEEE Transactions on Communications, Vol. 38, No. 9, September 1990

[6] Y. B. Lin, S. Mohan and A. Noerepel, "Queueing Priority Channel Assignment Strategies for PCS HandOff and Initial Access," IEEE Transactions on Vehicular Technology, Vol. 43, No. 3, August 1994.

[7] Everitt, D. E., "Traffic engineering of the radio interface for cellular mobile networks," Proc. IEEE, vol. 82, no. 9, pp. 1371-1382, 1994.

[8] A Comprehensive Call Management Strategy for Congestion Control in Cellular Networks by N.V.Marathe ,G.S.Biradar, U.B.Desai, S.N. Merchant

[9] H. Li and K. Yamanishi. Topic analysis using a finite mixture model. Inf. Process. Manage., 39(4):521-541, 2003.

[10] Topic Detection by Clustering Keywords Christian Wartena and Rogier Brussee Telematica Institute, 